\title{
Measurements of Plasma Termination in ICRF Heated Long Pulse Discharges with Fast Framing Cameras in the Large Helical Device*) $^{*}$
}

\author{
Mamoru SHOJI, Hiroshi KASAHARA, Hirohiko TANAKA, Tetsuo SEKI, Kenji SAITO, \\ Shuji KAMIO, Ryosuke SEKI, Takashi MUTOH, Masayuki TOKITANI, Shigeru MORITA, \\ Motoshi GOTO, Tetsutaro OISHI, Eduardo DE LA CAL ${ }^{1)}$, Carlos HIDALGO ${ }^{1)}$, \\ Hiroshi YAMADA and the LHD Experiment Group \\ National Institute for Fusion Science, 322-6 Oroshi-cho, Toki 509-5292, Japan \\ ${ }^{1)}$ Centro de Investigaciones Energéticas, Medioambientales y Tecnológicas, 40 Avenida Complutense, \\ Madrid 28040, Spain
}

(Received 21 November 2014 / Accepted 5 March 2015)

\begin{abstract}
The termination process of long pulse plasma discharges in the Large Helical Device (LHD) have been observed with fast framing cameras, which shows that the reason for the termination of the discharged has been changed with increased plasma heating power, improvements of plasma heating systems and change of the divertor configuration, etc. For long pulse discharges in FYs2010-2012, the main reason triggering the plasma termination was reduction of ICRF heating power with rise of iron ion emission due to electric breakdown in an ICRF antenna. In the experimental campaign in FY2013, the duration time of ICRF heated long pulse plasma discharges has been extended to about 48 minutes with a plasma heating power of $\sim 1.2 \mathrm{MW}$ and a line-averaged electron density of $\sim 1.2 \times 10^{19} \mathrm{~m}^{-3}$. The termination of the discharges was triggered by release of large amounts of carbon dusts from closed divertor regions, indicating that the control of dust formation in the divertor regions is indispensable for extending the duration time of long pulse discharges.
\end{abstract}

(c) 2015 The Japan Society of Plasma Science and Nuclear Fusion Research

Keywords: long pulse discharge, ICRF heating, dust, closed divertor, LHD

DOI: $10.1585 /$ pfr. 10.3402040

\section{Introduction}

Steady state plasma discharge operation (SSO) is required for future nuclear fusion reactors. The Large Helical Device (LHD) can sustain plasmas without the toroidal plasma current by the plasma confinement magnetic field produced by non-axisymmetric super-conducting coils [1]. Long pulse plasma discharges in the LHD can be a useful test stand for simulating the SSO for saturated wall conditions (no particle pumping on the vacuum wall) which is an equivalent situation in future nuclear fusion reactors.

The SSO has been tried in several super-conducting Tokamak devices [2,3]. However, the termination processes of long pulse discharges have not yet fully investigated. In the LHD, several fast framing cameras have been equipped, which is advantageous for identifying the reason for the termination of long pulse discharges in detail. Observations with the fast cameras show that the termination process has been changed with the increase in plasma heating power, improvements of plasma heating systems, gas fueling method, heat load dissipation technique, and change of the divertor configurations, etc. Thanks to the

author'se-mail: shoji@LHD.nifs.ac.jp

*) This article is based on the presentation at the 24th International Toki Conference (ITC24). above modifications and improvements, the duration time of the discharges have been extended to $\sim 48$ minutes under a condition of a heating power of $\sim 1.2 \mathrm{MW}$ and a lineaveraged electron density of $\sim 1.2 \times 10^{19} \mathrm{~m}^{-3}$ in the experimental campaign in FY(Fiscal Year)2013.

In this paper, the termination process of the discharges in the experimental campaigns in FYs2010-2012 and that in FY2013 is presented. In the last section, three possible measures for extending the duration time of the plasma discharges are proposed.

\section{Experimental Setup for Long Pulse Discharges}

In long pulse discharges, plasmas have been sustained with Ion Cyclotron Range of frequency Heating (ICH) and with Electron Cyclotron resonance Heating (ECH) [4]. Three different types of ICRF antennas have been installed in three toroidal positions (3.5, 4.5 and 7.5 ports). Poloidal Array (PA) antennas and HAnd Shake form (HAS) antennas have been equipped since FY2010 at 7.5 port and FY2011 at 3.5 port, respectively. Since FY2013, FieldAligned Impedance Transforming (FAIT) antennas have been installed in 4.5 port, which has contributed to stable 


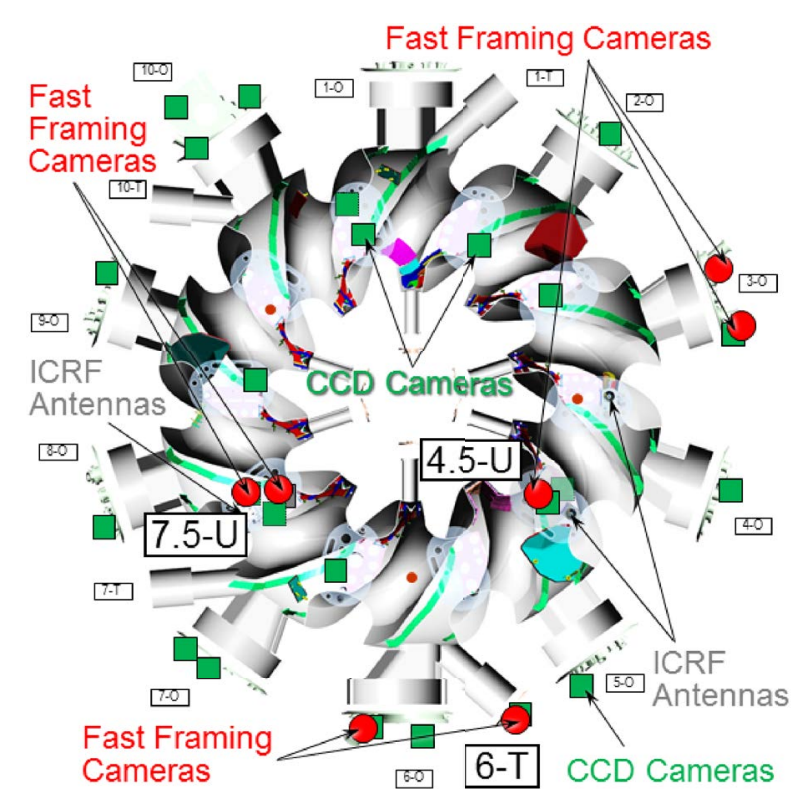

Fig. 1 Top view of the experimental setup of the one of plasma heating systems (ICRF antennas), visible CCD cameras (green squares) and fast framing cameras (red circles) for monitoring long pulse discharges in the LHD.

long pulse plasma discharges in FY2013.

For monitoring plasmas and the plasma-wall interactions in long pulse discharges, totally about thirty video rate (30 fps) visible CCD cameras are set in some viewports. Additionally, three visible fast framing cameras with bifurcated image fibers or stereo optics have been installed, which are mainly used for observing the termination process of long pulse discharges at high frame rates ( $\geq 1,000 \mathrm{fps})$. Figure 1 illustrates the top view of the experimental setup of the cameras and the plasma heating systems. Two optical lenses for a fast framing camera (Photron FASTCAM APX-RS) with a bifurcated image fiber or stereo optics have been set in two viewports (6$\mathrm{T}$ and 6-O) for monitoring the plasmas and ICRF antennas installed in 7.5 and 3.5 ports, respectively. Other fast framing camera (Photron FASTCAM SA-X) has been installed with two optical lenses and a bifurcated image fiber. The two lenses are set in the right and left side of an outer port (3-O) for stereoscopically observing the plasmas. Another fast camera having two separated camera heads (Photron FASTCAM MC2.1) has been equipped in an upper port 7.5-U (2 positions), or 4.5-U (1 position) for monitoring the ICRF antennas from the upper ports.

\section{Observations of the Termination Process of Long Pulse Discharges in FYs2011-2012}

Just before the plasma termination in long pulse discharges in FY2011-2012, the sparks in the ICRF antennas and the release of large amounts of dusts from the antennas were observed with the fast framing camera in the tangential port (6-T). The longest plasma duration time was limited by this phenomenon to 1,136 second under a condition of a heating power of $\sim 1.2 \mathrm{MW}(\mathrm{ICH}+\mathrm{ECH})$ and a line-averaged electron density of $\sim 1.0 \times 10^{19} \mathrm{~m}^{-3}$. A sequential image of the tangential view of the plasma and the ICRF antennas observed with the fast framing camera is shown in Fig. 2 with the time evolution of the plasma radiation power observed with an Absolute eXtreme UltraViolet photoDiode (AXUVD) [5], the intensity of iron and carbon ion emission, the line-averaged electron density, and the ICRF heating power. It indicates that the ICRF heating power from the 7.5-L antenna stopped by an interlock system which detects electric breakdown in the antenna at 1096.243 s. At this time, sparks in the antenna and the rise of iron ion emission were observed. A pre-programmed restart of the ICRF heating using this antenna was tried at $1096.458 \mathrm{~s}$ for recovering the heating power, which was failed by electric breakdown in the antenna. It could induce release of large amounts of dusts into the main plasma which was observed with the fast camera (Fig. 2 (b)). The abrupt increase in the iron ion emission at the first electric breakdown (1096.243 s) could be caused by release of iron-rich dusts from the antenna, which is composed of stainless steel, due to physical sputtering in the antenna. It should be noticed that the rise of the plasma radiation and the iron emission is earlier than that of the carbon emission by $\sim 0.1 \mathrm{~s}$, indicating that the plasma termination was not triggered by carbon. The above experimental results suggest that control of the electric breakdown in the antennas and suppression of dusts is essential for extending the plasma duration time.

\section{Observations of the Termination Process of Long Pulse Discharges in FY2013}

After the experimental campaign in FY2012, some modifications in the ICRF heating systems were taken for preventing electric breakdown in the antennas, such as tungsten coating on the antenna strips and removal of a part of Faraday shields, etc. In FY2013, most of divertor configuration was changed from an open divertor configuration to a closed one. The termination of long pulse discharges with the sparks in the antennas was significantly reduced. The longest duration time of the plasma discharges were extended to 2,860 seconds ( 48 minutes) under a condition of a heating power of $\sim 1.2 \mathrm{MW}$ (ICH+ECH), a central electron temperature of $\sim 2.0 \mathrm{keV}$ and a line-averaged electron density of $\sim 1.2 \times 10^{19} \mathrm{~m}^{-3}$. The long pulse plasma discharge was terminated with abrupt increase of carbon ion emission. Figure 3 is the typical time evolution of the plasma heating power by ICRF, and the plasma radiation and the intensity of impurity ion emission (iron and carbon) just before the plasma termination. Plasma heating power was almost constantly sustained until the end of the discharge, which is different from that in the experimental campaigns in FYs2010-2012. 
(a)

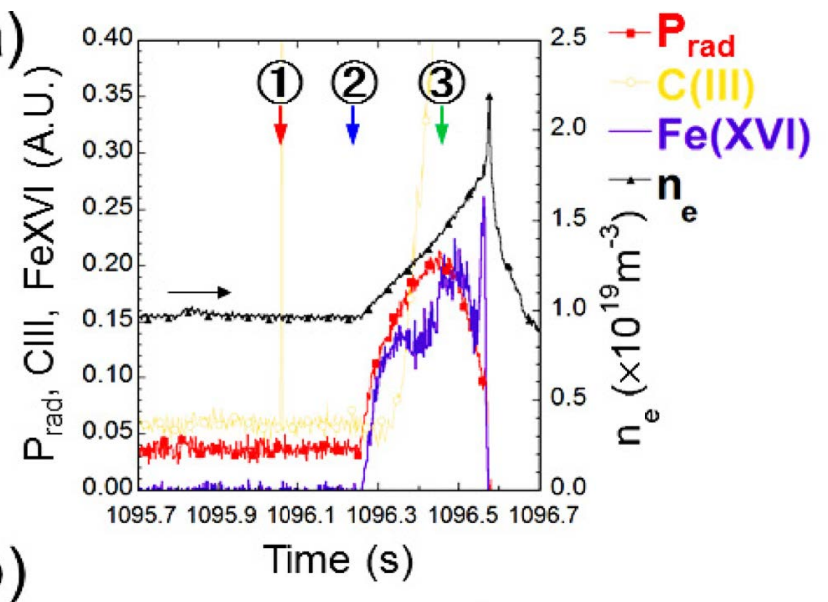

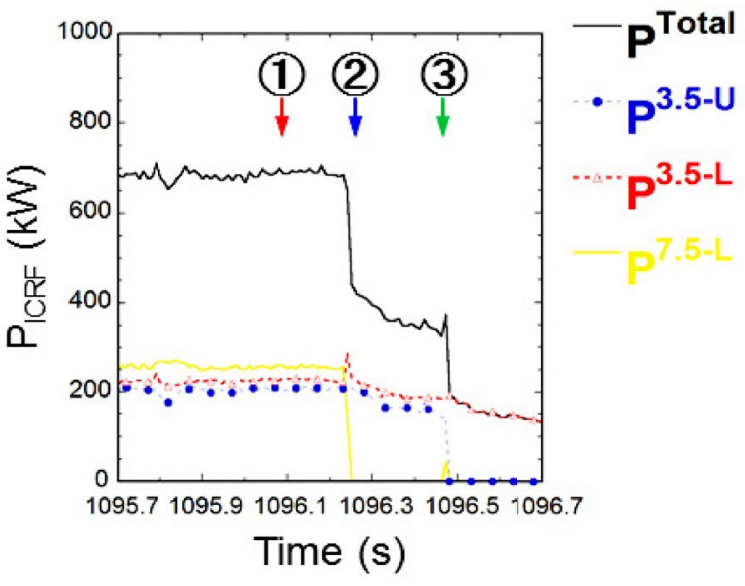
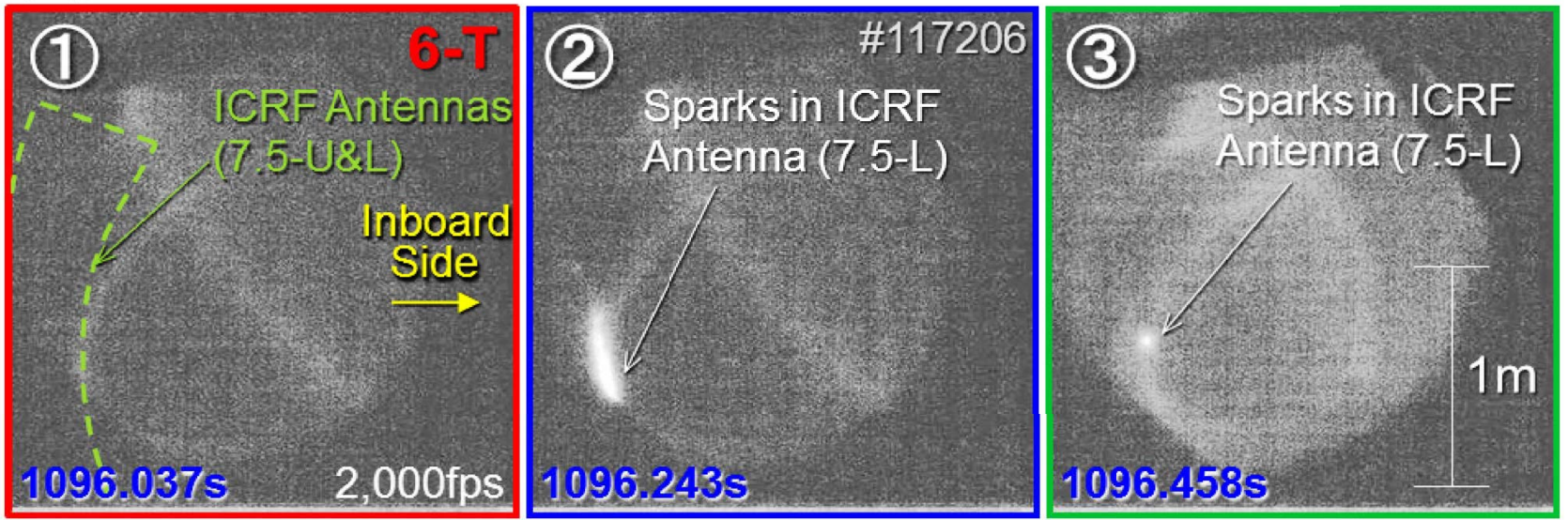

Fig. 2 (a) Time evolution of the plasma radiation, the intensity of iron and carbon ion emission, the line-averaged electron density and the ICRF heating power just before the plasma termination in an long pulse discharge in the experimental campaign in FY2012. (b) Sequential images of the tangential view of the plasma and ICRF antennas (7.5-U and L) observed with a fast framing camera in the 6-T port just before the plasma termination.

The rise of the plasma radiation and the impurity ion emission simultaneously occurred at $\sim 2860.35 \mathrm{~s}$. The increase in the iron ion emission is moderate compared to that in the carbon ion emission.

Release of large amounts of dusts was detected with the fast framing camera in $4.5-\mathrm{U}$ port just before the plasma termination. It shows that the dusts were released from a closed divertor region in the inboard side of the tours near a lower port (4.5-L) as shown in Fig. 4. After the experimental campaign, traces of exfoliation of carbonrich mixed material layers formed on brittle iron-rich layers deposited on the surface of closed divertor components (a dome structure and an inner vacuum vessel) was found on the site [6]. In the closed divertor configuration, some divertor plates (isotropic carbon) near the lower port are arranged for blocking the plasmas in the divertor legs in order to prevent neutral particles from escaping from the divertor region through the edge of the divertor components [7]. It is probable that the divertor plates, which face to the inboard side of the tours, enhance the deposition layers on the divertor components by physical and chemical sputtering on the plates.

Other plasma termination process was found with the

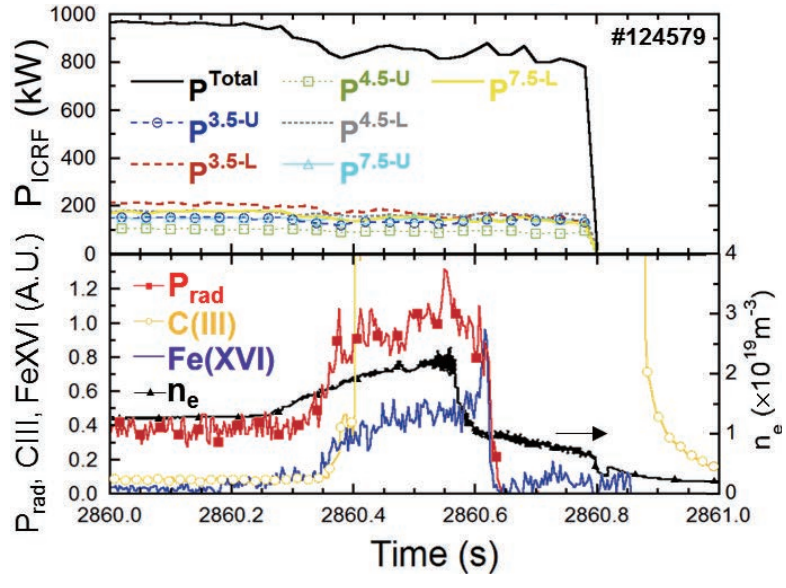

Fig. 3 Time evolution of the plasma heating power by ICRF, the line-averaged plasma density, the radiation power, and the intensity of impurity ion emission (iron and carbon) just before the termination of a long pulse plasma discharge in the experimental campaign in FY2013.

fast framing camera in the tangential port (6-T) in a long pulse discharge with a high heating power of $\sim 3 \mathrm{MW}$. Figure 5 shows sequential images of the tangential view ob- 

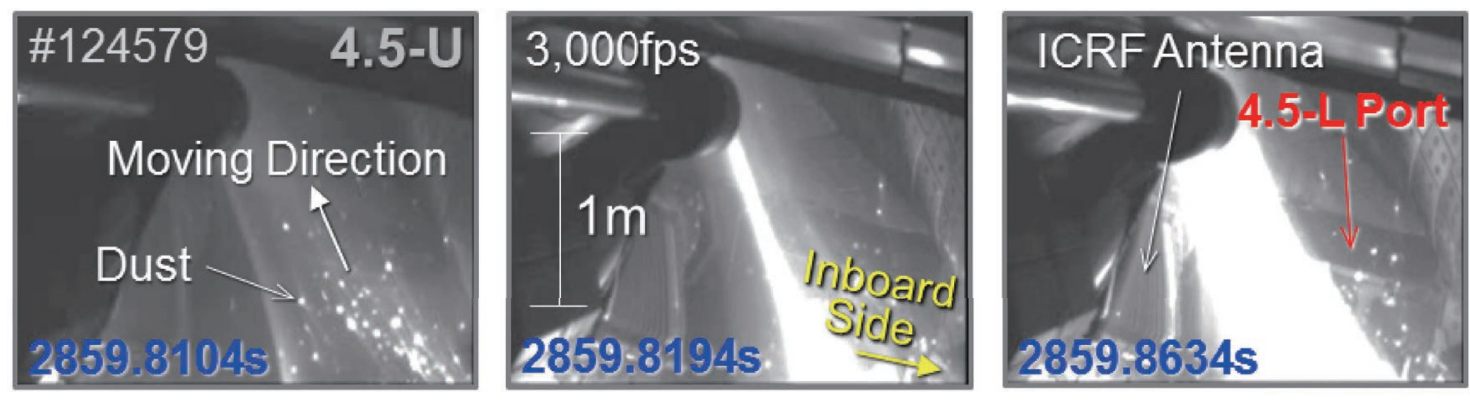

Fig. 4 Sequential images observed with a fast framing camera installed in an upper port (4.5-U) just before the termination of a long pulse plasma discharge in FY2013. Note that the elapsed time in this figure can be different from that in Fig. 3 because of the different time counter system for the plasma diagnostics.
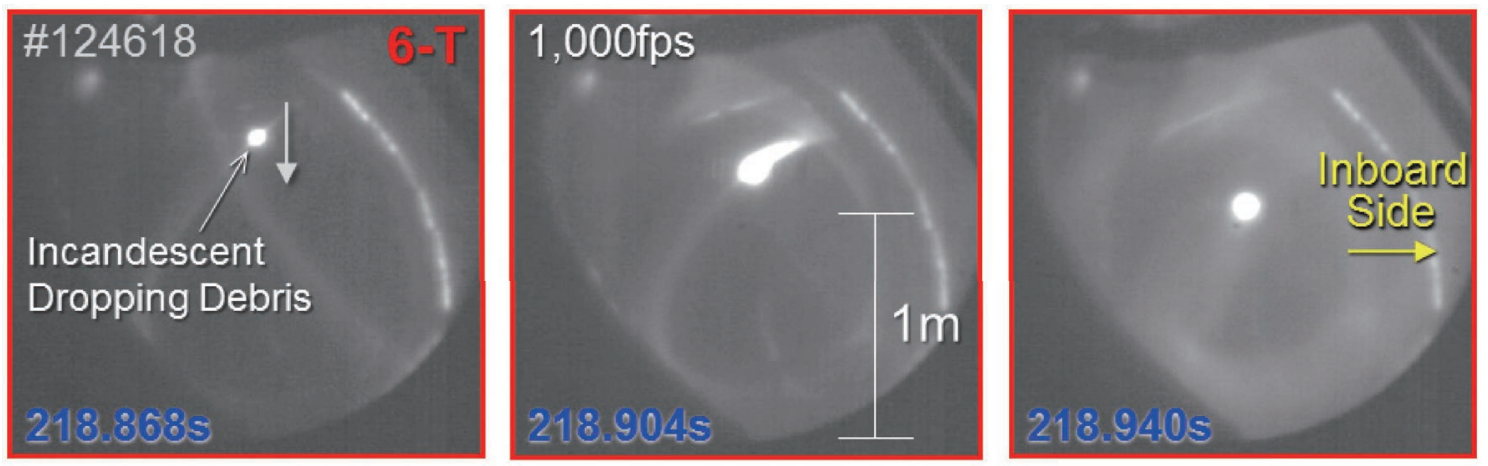

Fig. 5 Sequential images of a tangential view of the plasma just before plasma termination in a long pulse discharge with a high ICRF heating power of $\sim 3 \mathrm{MW}$ in FY2013.

served just before the plasma termination. It shows that an incandescent debris, which dropped from a position near an upper port (8.5-U), terminated the plasma discharge. After the experimental campaign, a melted head of a stainless steel bolt was found on a support structure for divertor plates near the upper port locating on armor tiles covering the vacuum vessel. The melted position was apart from divertor plates to a helical coil side, suggesting that high energy protons accelerated just in front of the ICRF antennas in 7.5-U port directly struck the support structure [8], which could lead to the drop of the head of the bolt.

The above observations with the fast framing camera propose the following possible measures for extending the duration time of long pulse plasma discharges in the LHD:

1. Modification of the closed divertor configuration near the lower ports for reducing the deposition layers on the dome structure and the inner vacuum vessel,

2. Intense conditioning in the closed divertor region near the lower ports before long pulse discharge experiments by adopting a special magnetic configurations in which the strike points are on the positions where the deposition layers are formed,

3. Protection of the support structure for the divertor plates at the position where the high energy protons accelerated just in front of the ICRF antennas directly hit by using extended divertor plates.

\section{Summary}

The termination processes of long pulse discharges in the LHD have been observed with the fast framing cameras. It shows that the stop of the ICRF heating power and the release of iron dusts by electric breakdown in the antenna are the main reason for triggering the termination of the long pulse discharges in the experimental campaigns in FYs2010-2012. In FY2013, while the termination of long pulse discharges with the sparks in the antennas was significantly reduced, release of large amounts of carbon dusts from the closed divertor region near a lower port is the main cause to trigger the plasma termination. At the termination of a high heating power discharge, a drop of a melted bolt heat was observed at the position where high energy protons accelerated by ICRF just in front of the antennas hits. Investigation of the observations with the fast framing cameras proposed three possible measures for extending the duration time of long pulse discharges.

\section{Acknowledgments}

This work is financially supported by NIFSULPP015 and by NIFS/NINS under the project of Formation of International Scientific Base and Network with an international collaboration research framework between NIFS and CIEMAT in Spain. 
[1] A. Komori et al., Fusion Sci. Technol. 58, 1 (2010).

[2] D. Vanhoutte et al., Nucl. Fusion 44, L11 (2004).

[3] M. Sakamoto et al., Nucl. Fusion 44, 693 (2004).

[4] T. Mutoh et al., Nucl. Fusion 53, 063017 (2013).

[5] B. Peterson et al., Plasma Phys. Control. Fusion 45, 1167
(2003).

[6] M. Tokitani et al., J. Nucl. Mater. (to be published).

[7] T. Morisaki et al., Nucl. Fusion 53, 063014 (2013).

[8] M. Shoji et al., J. Nucl. Mater. 337-339, 186 (2005). 